\title{
Epigallocatechin 3-gallate Binds to Human Salivary $\alpha$-Amylase with Complex Hydrogen Bonding Interactions
}

\author{
Jee-Young Lee, Ki-Woong Jeong, and Yangmee Kim* \\ Department of Bioscience and Biotechnology, Bio/Molecular Informatics Center, Konkuk University, Seoul 143-701, Korea \\ *E-mail:ymkim@konkuk.ac.kr
}

Received April 27, 2011, Accepted May 16, 2011

\begin{abstract}
Amylase is a digestive enzyme that catalyses the starch into sugar. It has been reported that the green tea flavonoid (or polyphenols) (-)-epigallocatechin 3-gallate (EGCG) inhibits human salivary $\alpha$-amylase (HSA) and induced anti-nutritional effects. In this study, we performed docking study for seven EGCG-like flavonoids and HSA to understand the interaction mechanism of HSA and EGCG and suggest new possible flavonoid inhibitors of HSA. As a result, EGCG and (-)-epicatechin gallate (ECG) bind to HSA with complex hydrogen bonding interactions. These hydrogen bonding interactions are important for inhibitory activity of EGCG against HSA. We suggested that ECG can be a potent inhibitor of HSA. This study will be helpful to understand the mechanism of inhibition of HSA by EGCG and give insights to develop therapeutic strategies against diabetes.
\end{abstract}

Key Words : EGCG, EGC, Amylase, Flavonoids, Docking

\section{Introduction}

Amylase is a digestive enzyme that catalyses the starch into sugar. ${ }^{1,2}$ In nature, three types of amylase $(\alpha-, \beta$-, and $\gamma$ amylase) are present that $\alpha$-amylase exists in plant and animals; $\beta$-amylase is present in microbes. ${ }^{1,3,4} \alpha$-Amylase ( $\alpha$-1,4-glucan-4-glucanohydrolase) catalyzes the hydrolysis of the $\alpha-(1,4)$-glycosidic linkages of starch components, glycogen and various oligosaccharides. Two different $\alpha$ amylase isoforms are present in human which are synthesized in the pancreas and the salivary glands, but the amylase in the salivary is the most abundant enzyme. ${ }^{2}$ Human pancreas $\alpha$-amylase (HPA) hydrolyzes the dietary starch into disaccharides and trisaccharides while human salivary $\alpha$ amylase (HSA) also degrades polymeric starch into shorter oligomers. ${ }^{2,5}$ The initial digestion of starch is started by HSA in the mouth and additional digestion is carried out by HPA..$^{2-4}$ In the case of diabetic patients, the elevation level of blood glucose after a meal presents a challenge for managing hyperglycemia. Therefore, the inhibition of $\alpha$-amylase is important to therapeutic strategy of diabetes. ${ }^{5}$

Both amylases are composed of 496 amino acids in a single polypeptide chain and they show a high degree of sequence similarity about $92 \% .^{2} \alpha$-Amylase is calciumbinding protein with a single polypeptide chain folded into three domains (A, B, and C domain). ${ }^{2,5}$ Domain A which is the largest catalytic domain has a $(\beta / \alpha)$ barrel structure and the inhibitor binding site is located in the middle of the domain A. A chloride ion is included in this domain. Domain B has no definite topology, but the calcium ion bound to domain B. These ions are not directly participated in substrate binding, but related on the structural stability of amylase and help the binding of ligand in active site. Domain $\mathrm{C}$ has a Greek-key barrel structure. The 3D structure of HSA is depicted in Figure 1(a). ${ }^{2}$

(-)-Epigallocatechin 3-gallate (EGCG) is a major catechin in green tea and noted for its various biological effects. ${ }^{6}$ The antioxidative effect and antitumor effect of EGCG is the most well known activities. ${ }^{7-10}$ It has been known that EGCG inhibits the biological activity of digestive enzymes such as lactase, pepsin, lipase, and amylase with their potential control of obesity, diabetes, and anti-nutritional effects. ${ }^{11,12}$ Among these, EGCG inhibits HSA stronger than the other digestive enzymes. ${ }^{11}$

In order to understand the interaction of HSA and EGCG, automated docking study was performed and a docking model between HSA and EGCG was proposed. From this study, we suggested possible natural products as inhibitors of HSA.

\section{Methods}

Docking Study. Docking model between HSA and EGCG was determined by AutoDock. ${ }^{13-15}$ The X-ray co-crystal structures of inhibitor, acarviostatin analog (A103), bound to HPA has been reported. ${ }^{5}$ Since the co-complex structure of substrate and HSA has not been determined, we adapted the binding interactions of inhibitor-HSA based on the x-ray cocomplex structures of HPA (3OLD.pdb). ${ }^{5}$ The interaction model of HPA-inhibitor (A103) is depicted in Figure 1(b). We utilized X-ray structure of HSA which deposited in the Protein Data Bank (1SMD.pdb and 1XV8.pdb). ${ }^{2}$ The Lamarckian Genetic Algorithm (LGA) of the AutoDock 3.05 was used for docking experiments. MD simulations on the final docking structure were performed in the canonical ensemble (NVT) at $300 \mathrm{~K}$ and distance-dependent function of the dielectric constant was used for the calculation of the energetic maps in vacuum system using the program InsightII/ 
Discover. All atoms of the system were considered explicitly, and their interactions were computed using the consistent valence force field. A distance cutoff of $10 \AA$ was used for van der Waals interactions and electrostatic interactions. The time step in the MD simulations was $1 \mathrm{fs}$ and $\mathrm{MD}$ simulation was performed for $2 \mathrm{~ns}$. Coordinates were saved every $1 \mathrm{ps}$. The average structure was calculated for the 2 ns trajectory and submitted to final energy minimization by performing 10,000 steps of steepest descent method.

We collected the structure of seven catechin based polyphenolic flavonoids including EGCG extracted from green and black tea and performed docking study. The structures of polyphenolic flavonoids are shown in Figure 2.

\section{Results and Discussion}

Analysis of the X-ray Co-Crystal Structure of HPAInhibitor Complex. The X-ray co-crystal structure of inhibitor, acarviostatin analog (A103), bound to HPA have been proved while there is no information about interactions between HSA and inhibitor available, yet. Acarviostatin family is secondary metabolites secreted by Streptomyces coelicoflavu and pseudo-oligosaccharides containing several acarviosineglucose units and a varying number of glucose residues. ${ }^{5}$ It is the most potent inhibitor of HPA to date. A103 is a pseudohexasaccharide composed of an acarviosine-glucose unit bound to maltotriose. The inhibition of HPA by A103 is depends on complex hydrogen bonds between the hydroxyl groups of the inhibitor and the catalytic residues of the binding site. As shown in Figure 1(b), the many ribose hydroxyl groups of A103 participated in hydrogen bonding interactions with catalytic residues of HPA in active site including side chains of Q63, H101, D197, R195, K200, H201, E233, E240, H299, D300, and H305, as well as backbone oxygens of T63, W58, and G306. These hydrogen bonding interactions contribute to tight binding of A103 to HPA. The aromatic hydrophobic residues, W58, Y62, and Y151, form hydrophobic site, too. ${ }^{5}$

Docking Results of HSA and Flavonoids. We performed docking with HSA and seven catechin family polyphenols including EGCG. Two polyphenols, EGCG and (-)-epicatechin gallate (ECG) were hit flavonoids and docked well into the active site of HSA.

The docking model of HSA-EGCG is composed of various hydrogen bonding interactions. Structure of EGCG is divided into three chemical groups; catechin (A-C ring), epigallo (B-ring), and gallate (D-ring) moieties, as shown in Figure 2. The three hydroxyl groups of epigallo moiety formed complex hydrogen bonding interactions with side chains of R195, H299, E231, E233, and D300, and hydrophobic interactions with W58 and Y62. The two hydroxyl groups of catechin moiety formed hydrogen bonding interactions with side chain of $\mathrm{H} 201$ and the backbone carboxyl oxygen of G306, respectively. Catechin moiety was also bound to V234 and I235. The gallate moiety has three hydroxyl groups and two hydroxyl groups participated in hydrogen bondings with backbone carboxyl oxygen of Y62 (a)
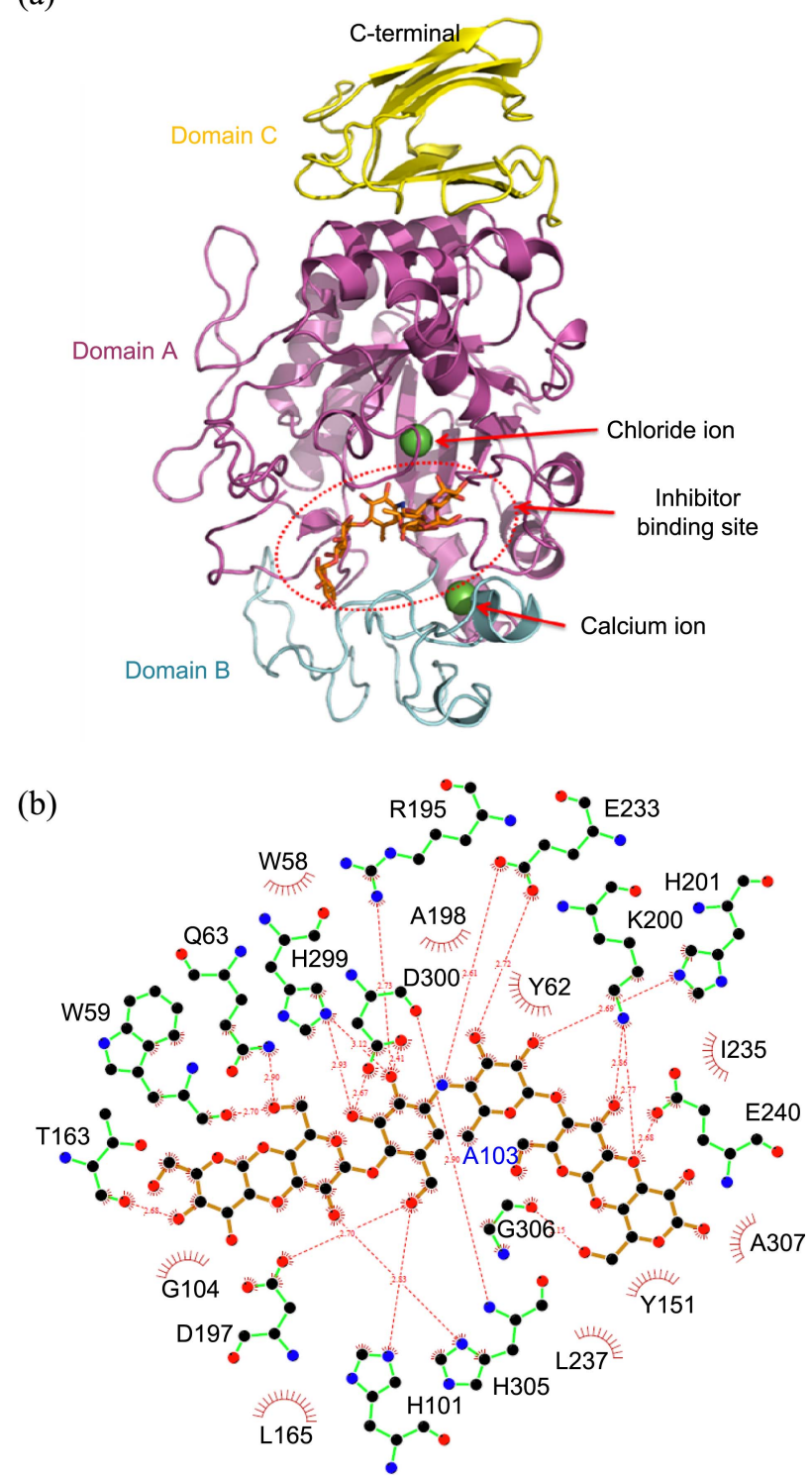

Figure 1. The structure of HSA and the interaction model between HPA and inhibitor, A103. (a) The 3D structure of HSA. (b) Interaction model between HPA-A103 (3OLD.pdb) represented using Ligplot. The plot shows the complex hydrogen bonding interactions. Hydrogen bonds are displayed using dashed lines while hydrophobic interactions are represented by an arc with spokes radiating towards the ligand atoms.

and side chain of Q63, respectively. Y59, L162, L165, and T163 formed hydrophobic interaction with gallate moiety. The hydrogen bondings and hydrophobic interactions of HSA-EGCG showed similar pattern to those of HPA-A103. Since the size and the number of hydroxyl groups of EGCG are smaller than those of A103, hydrogen bonding interactions of HSA-EGCG were much simpler compared with those of HSA-A103. The interaction model between HSA and EGCG is shown in Figure 3(a). From docking study, we confirmed that HSA and EGCG formed specific and tight hydrogen bonding interactions.

ECG is a flavan-3-ol, a type of flavonoid, present in green tea and grape ${ }^{16}$ The whole structure of ECG is equal to 
<smiles>CCOc1cc(O)cc2c1C[C@@H](OC(=O)c1cc(O)c(O)c(O)c1)[C@H](c1cc(O)c(O)c(O)c1)O2</smiles>

(-)-Epigallocatechin gallate (EGCG)<smiles>O=C(O[C@H]1Cc2c(O)cc(O)cc2OC1c1cc(O)c(O)c(O)c1)c1cc(O)c(O)c(O)c1</smiles>

(+)-Gallocatechin-3-gallate (GCG)<smiles>O=C(O[C@H]1Cc2c(O)cc(O)cc2O[C@H]1c1ccc(O)c(O)c1)c1cc(O)c(O)c(O)c1</smiles>

(-)-Epicatechin gallate (ECG)

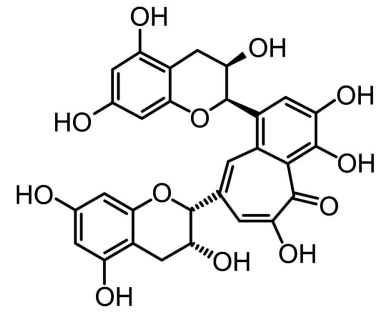

Theaflavin<smiles>O=C(OC1Cc2c(O)cc(O)cc2OC1c1cc(C2Oc3cc(O)cc(O)c3C[C@H]2O)cc(O)c2c(O)c(=O)c(O)cc1-2)c1cc(O)c(O)c(O)c1</smiles>

Theaflavin-3-gallate

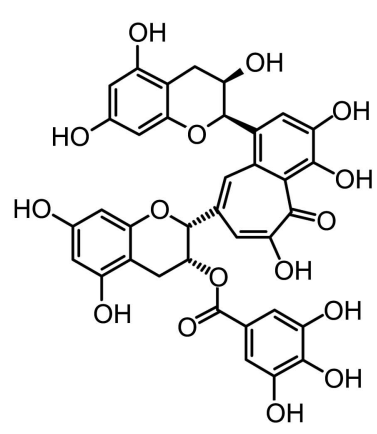

Theafkavin-3'-gallate<smiles></smiles>

Theaflavin-3,3'-gallate

Figure 2. Chemical structures of seven catechin based flavonoids.

(a)

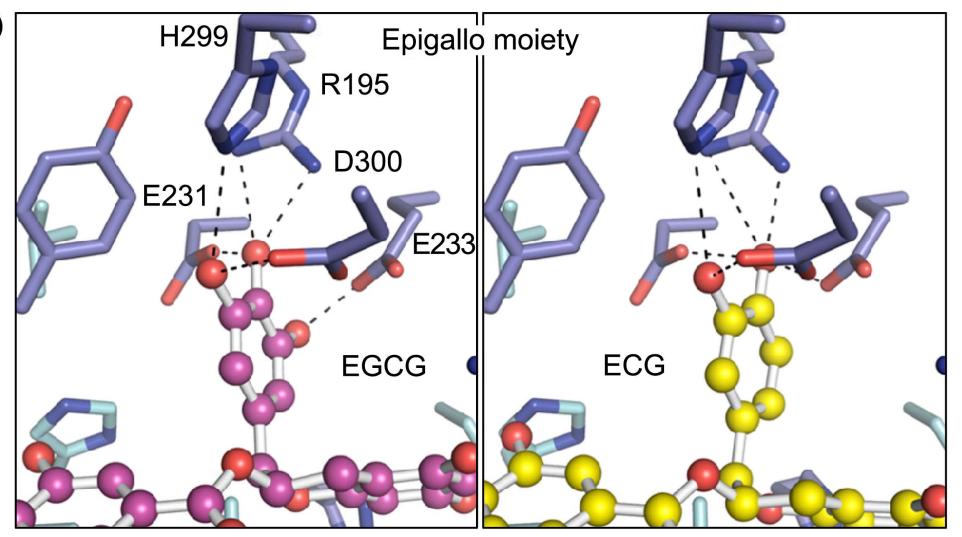

(b)
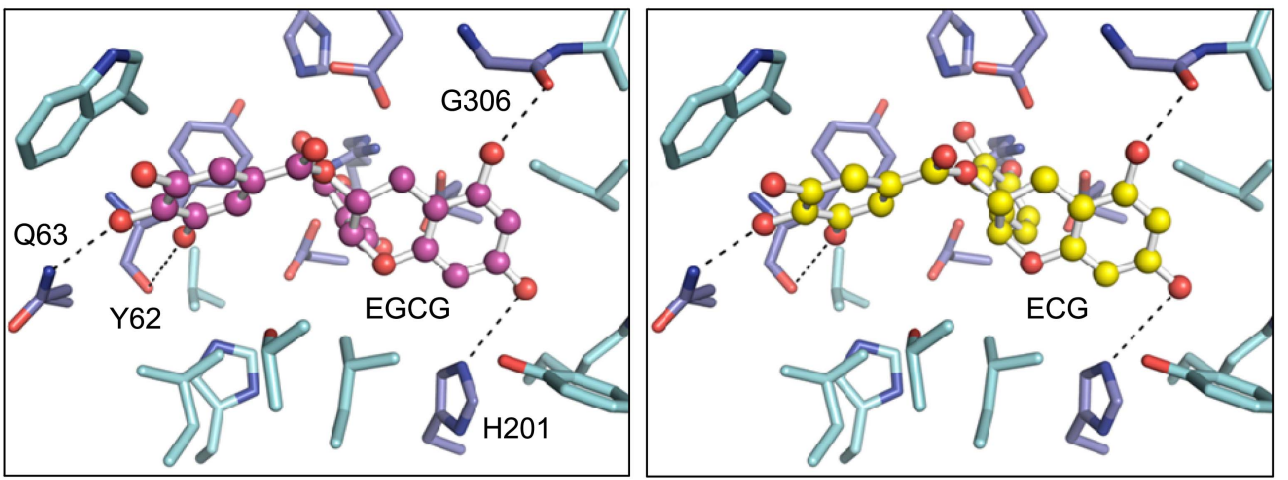

Figure 3. Docking model of flavonoids and HSA. (a) Interaction model of HSA and epigallo moiety of two flavonoids. (b) Interaction model of HSA and catechin-gallate moiety of flavonoids. EGCG and EGC formed identical hydrogen bonding interactions with HSA. 


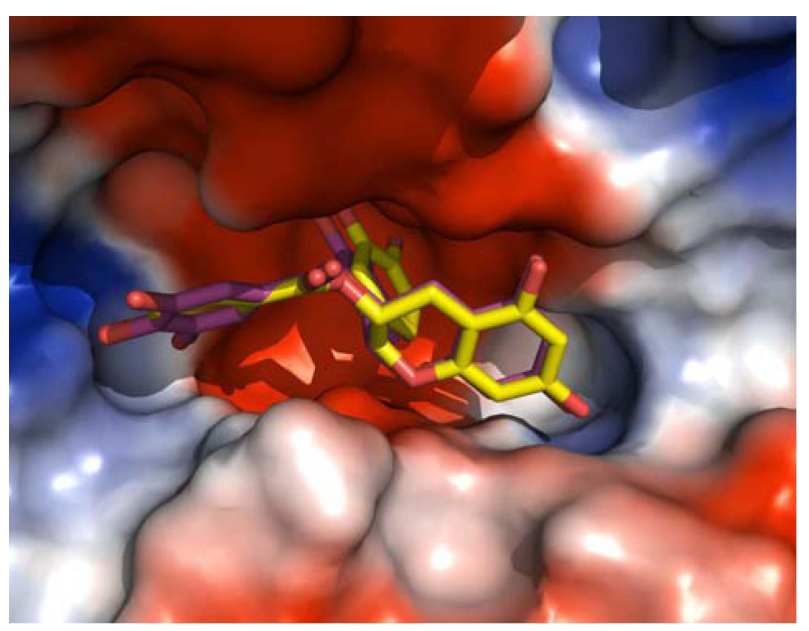

Figure 4. Superimposed surface models of HSA-EGCG and HSAEGC.

EGCG except that only one hydroxyl group is less than EGCG in epigallo moiety. Even though ECG has only two hydroxyl groups in epigallo moiety, the hydrogen bonding interactions with HSA were equal to those of HSA-EGCG. The interaction model between HSA and EGCG is shown in Figure 3(b) and the superimposed model of HSA-EGCG and HSA-EGC is depicted Figure 4.

$(+)$-Gallocatechin gallate (GCG) is composed the ester of gallocatechin and gallic acid and a type of catechin. ${ }^{17}$ Since it is an epimer of EGCG, its conformation could not be specified at the active site of HSA. The rest of five theaflavin family flavonoids were too large to dock into the active site of HSA. ${ }^{18,19}$

In order to predict the binding affinity of EGCG and EGC to HSA, we calculated ligand scoring (LigScore). LigScore is a scoring function that possesses high predictive accuracy of affinity of ligand-receptor binding as well as $\mathrm{p} K_{\mathrm{i}}$ values. ${ }^{20}$ LigScore of EGCG and EGC were 5.74 and 5.25, respectively. This implied that EGCG and EGC may bind to HSA with similar affinities and EGC can be a potent inhibitor of HAS, too.

From this study, we proposed that EGCG binds to HSA with complex hydrogen bonding interactions and these interactions play the major factors in its inhibitory activity against HSA. Also, we suggested that EGC can be a potent inhibitor of HSA the same as EGCG.

\section{Conclusion}

From automated docking study, we suggested a docking pose between HSA and EGCG. The docking model of HSAEGCG formed complex hydrogen bonding interactions. The three hydroxyl groups of epigallo moiety of EGCG formed various hydrogen bonding interactions with side chain nitrogens of R195, H299 and carbonyl oxygens of E231, E233, and D300. It formed hydrophobic interactions with W58 and Y62. The catechin moiety formed hydrogen bonding interactions with side chain of $\mathrm{H} 201$ and the backbone carboxyl oxygen of G306. Catechin moiety was also bound to V234 and 1235 which formed the hydrophobic environment. The two hydroxyl groups of gallate moiety participated in hydrogen bondings with backbone carboxyl oxygen of Y62 and side chain of Q63, respectively. Four hydrophobic residues including Y59, L162, L165, and T163 formed hydrophobic interactions with gallate moiety. Since A103 is much larger and has more hydroxyl groups than EGCG, hydrogen bonding interactions of HPA-A103 are more complex than those of HSA-EGCG. Another catechin flavonoid, ECG also docked well into the active site of HSA with identical hydrogen bonding interactions appeared in HSA-EGCG model. LigScore of EGCG for HSA was very similar to that of EGC, too. Therefore, we suggested that EGC can be an inhibitor of HSA with similar efficacy compared with EGCG. This study may provide an understanding of the interaction mechanism of EGCG with HSA for the development of novel HSA inhibitors. Further experimental study will be carried out to identify these HSA inhibitors in an attempt to develop therapeutic strategies against diabetes.

Acknowledgments. This work was supported by Priority Research Centers Program through the National Research Foundation of Korea (NRF) funded by the Ministry of Education, Science and Technology (2009-0093824) and by National Research Foundation of Korea (NRF) funded by the Ministry of Education, Science and Technology (20090076064). Ki-Woong Jung is supported, in part, by the second BK21 (MOE).

\section{References}

1. Park, K. H.; Kim, T. J.; Cheong, T. K.; Kim, J. W.; Oh, B. H.; Svensson, B. Biochim. Biophys. Acta 2000, 1478, 165.

2. Ramasubbu, N.; Paloth, V.; Luo, Y.; Brayer, G. D.; Levine, M. J. Acta. Crystallogr. D Biol. Crystallogr. 1996, 52, 435.

3. Christiansen, C.; Abou Hachem, M.; Janecek, S.; Viksø-Nielsen, A.; Blennow, A.; Svensson, B. FEBS J. 2009, 276, 5006.

4. Sun, H.; Zhao, P.; Ge, X.; Xia, Y.; Hao, Z.; Liu, J.; Peng, M. Appl. Biochem. Biotechnol. 2010, 160, 988.

5. Qin, X.; Ren, L.; Yang, X.; Bai, F.; Wang, L.; Geng, P.; Bai, G.; Shen, Y. J. Struct. Biol. 2011, 174, 196.

6. Chen, D.; Milacic, V.; Chen, M. S.; Wan, S. B.; Lam, W. H.; Huo, C.; Landis-Piwowar, K. R.; Cui, Q. C.; Wali, A.; Chan, T. H.; Dou, Q. P. Histol. Histopathol. 2008, 23, 487.

7. Katiyar, S. K.; Matsui, M. S.; Elmets, C. A.; Mukhtar, H. Photochem. Photobiol. 1999, 69, 148.

8. Xu, Z.; Chen, S.; Li, X.; Luo, G.; Li, L.; Le, W. Neurochem. Res. 2006, 31, 1263.

9. Qanungo, S.; Das, M.; Haldar, S.; Basu, A. Carcinogenesis 2005, 26,958 .

10. Sue, Brierley-Hobson. Bioscience Horizons 2008, 1, 9.

11. He, Q.; Lv, Y.; Yao, K. Food Chem. 2006, 101, 1178.

12. Naz, S.; Siddiqi, R.; Dew, T. P.; Williamson, G. J. Agric. Food Chem. 2011, 59, 2734.

13. Lee, J. Y.; Lee, S. A.; Kim, Y. Bull. Korean Chem. Soc. 2007, 28, 941.

14. Lee, J. Y.; Baek, S.; Kim, Y. Bull. Korean Chem. Soc. 2007, 28, 379.

15. Morris, G. M.; Goodsell, D. S.; Halliday, R. S.; Huey, R.; Hart, W. E.; Belew, R. K.; Olson, A. J. J. Computational Chemistry 1998, 19, 1639.

16. Shiota, S.; Shimizu, M.; Mizushima, T.; Ito, H.; Hatano, T.; 
Yoshida, T.; Tsuchiya, T. Biol. Pharm. Bull. 1999, 22, 1388.

17. Ikeda, I.; Kobayashi, M.; Hamada, T.; Tsuda, K.; Goto, H.; Imaizumi, K.; Nozawa, A.; Sugimoto, A.; Kakuda, T. J. Agric. Food Chem. 2003, 51, 7303.

18. Khan, N.; Afaq, F.; Saleem, M.; Ahmad, N.; Mukhtar, H. Cancer
Res. 2006, 66, 2500.

19. Chung, J. Y.; Huang, C.; Meng, X.; Dong, Z.; Yang, C. S. Cancer Res. 1999, 59, 4610.

20. Krammer, A.; Kirchhoff, P. D.; Venkatachalam, X. J. C. M.; Waldman, M. J. Mol. Graph. Model. 2005, 23, 395. 